\title{
Self-Similar Multiresonant Nanoantenna Arrays for Sensing from Near- to Mid-Infrared
}

\author{
Daniel Rodrigo, ${ }^{\dagger, \ddagger \S}$ Andreas Tittl, ${ }^{\dagger, \S}$ Aurelian John-Herpin, ${ }^{\dagger}$ Odeta Limaj, ${ }^{\dagger}$ and Hatice Altug ${ }^{*},{ }^{\dagger}$ \\ ${ }^{\dagger}$ Institute of Bioengineering, École Polytechnique Fédérale de Lausanne (EPFL), Lausanne 1015, Switzerland \\ ${ }^{\ddagger}$ ICFO, Institut de Ciències Fotòniques, The Barcelona Institute of Science and Technology, 08860 Castelldefels, Spain
}

\section{Supporting Information}

ABSTRACT: Tailoring nanoscale light concentration and electromagnetic near-field enhancement over a broad spectral range is crucial for many photonics applications such as infrared spectroscopy, photodetection, and light harvesting. So far, broadband light enhancement has faced significant challenges due to the difficulty of efficiently exciting resonances at spectrally separated wavelengths and the inability of current devices to individually tune each specific resonance. Here, we introduce a multiresonant structure based on the non-overlapping combination of plasmonic nanoantenna arrays with multiple periodicities. The self-similarity of the multiperiodic array, obtained by a fractal-like generation procedure, enables the excitation of a high number of

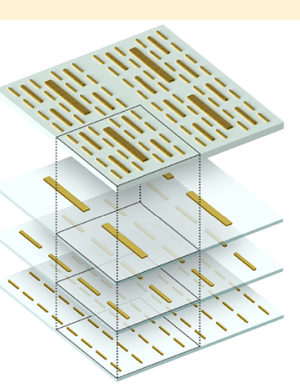

Frequency $\left(\mathrm{cm}^{-1}\right)$

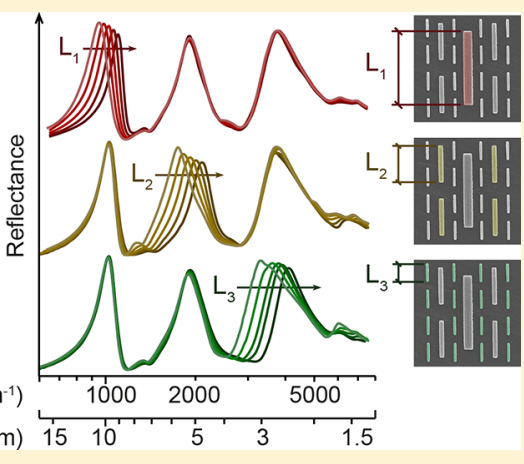
resonances without compromising their excitation efficiency. We experimentally demonstrate devices with up to four independent resonances covering an unprecedentedly wide spectral range from 10 to $1.5 \mu \mathrm{m}$. Significantly, the reflectance signal is uniformly strong for all the resonances, reaching more than $70 \%$ amplitude and near-field intensity enhancements above 1000 . We further show that each individual resonance wavelength can be independently controlled over a $50 \%$ spectral range by modifying a single geometrical antenna parameter, providing superior flexibility in tailoring the overall spectral response. Due to the self-similar layout and independent resonances, our design is well described by temporal coupled-mode theory, allowing for a straightforward extension for other nanophotonic applications. Finally, we demonstrate that the wide spectral coverage of our design enables a unique sensing method by simultaneously performing chemically specific mid-infrared detection and nearinfrared refractometry.

KEYWORDS: nanoantennas, plasmonics, multiresonant, broadband, surface-enhanced infrared spectroscopy, biosensors

M etallic nanoantennas are currently the focus of broad scientific interest due to their unique ability to collect free-space propagating light and confine it into subwavelength volumes, where extreme enhancements of electromagnetic fields are produced. ${ }^{1,2}$ Such plasmonic nanostructures and nanophotonic concepts provide new routes toward light manipulation at the nanoscale ${ }^{3,4}$ and have led to innovations in a wide range of applications such as sensing, ${ }^{5-7}$ spectroscopy, ${ }^{8-10}$ photovoltaics, ${ }^{11,12}$ planar optics, ${ }^{13}$ catalysis, ${ }^{14}$ or photodetection. $^{15}$

A variety of optical nanoantenna designs have been implemented to realize a multitude of applications over different wavelength ranges from terahertz to the ultraviolet. However, the spectral range of operation of a typical nanoantenna geometry is constrained by its resonant nature, which limits its light enhancement ability to a comparatively narrow bandwidth. ${ }^{16}$ This limitation hampers a wide range of nanophotonic applications. For instance, single-resonance optical nanoantennas limit the collected wavelength range from a broad solar spectrum in photovoltaics. Likewise, they impede multifunctional optical platforms that can accommo- date different applications into a single-device such as the combination of surface-enhanced Raman spectroscopy in the near-infrared with absorption spectroscopy in the infrared. ${ }^{17,18}$ In infrared (IR) spectroscopy and sensing, a single resonant nanoantenna cannot access the full set of molecular absorption fingerprints and therefore provides only a partial view of the complete chemical information available in the infrared spectrum. ${ }^{19,20}$ Thus, there is an increasing need to conceptualize and develop novel nanostructures with wider bandwidth that can manipulate and enhance light over extended spectral ranges.

In the pursuit of broadband light enhancement, various multiresonant plasmonic nanoantenna structures are currently under study, including fractal geometries, ${ }^{21-25} \mathrm{cross}^{26,27}$ and U-T shapes, ${ }^{28,29}$ superimposed Moire ${ }^{30}$ and Fisher ${ }^{31}$ patterns, concentric nanorings, ${ }^{32}$ multigratings, ${ }^{33,34}$ polarization-dependent antennas, ${ }^{3,36}$ zigzag trapezoids, ${ }^{37,38}$ and tapered multidipoles. ${ }^{39,40}$ The common idea behind these methods is

Received: July 30, 2018

Published: October 3, 2018 
the use of multiscale geometries that allow the excitation of multiple resonances. However, in the design of multiresonant antennas it is fundamentally challenging to accommodate resonant modes with equally strong excitation efficiencies over a broad spectrum due to the large size mismatch between the antennas resonating at the far end points of the spectral operating range. In particular, the high-frequency modes are typically weak, strongly limiting their usability. Additionally, the large nanoantenna array periodicities needed to support operation at long-wavelengths can produce unwanted excitation of grating modes at shorter wavelengths. As a result, current multiresonant nanoantennas allow only a small number of resonant modes with nonuniform spectral coverage. Furthermore, some of the introduced plasmonic designs are based on complex unit cell geometries where scaling and nanofabrication are not straightforward. In such cases, the specific resonance wavelengths are mutually interdependent and often cannot be independently controlled in a simple manner.

In this paper, we present a novel technique to produce highly efficient and versatile multiresonant structures using multiscale self-similar arrays. In contrast to existing works, the present approach realizes multiple resonances by combining independent subarrays consisting of different plasmonic nanoantenna sets where each of them provides a distinct resonance frequency. The novel feature of our technique is that each subarray is implemented by applying a scaling factor that affects not only the antenna dimensions, but also the periodicity of the array. The structure is designed following a fractal-like generation procedure that allows the nonoverlapping superposition of the subarrays and their corresponding spectral responses. The main advantage of our technique arises from scaling the periodicities of the subarrays, thus increasing the number of active elements in higher frequency resonances and preventing the excitation of higher order grating modes. We experimentally demonstrate devices with up to four independent well-defined resonances covering an unprecedentedly wide spectral range from mid-IR to near-IR wavelengths $(10-1.5 \mu \mathrm{m})$. Significantly, the resonancemediated reflectance is equally strong for all resonances, reaching more than $>70 \%$ amplitude with associated near-field intensity enhancements above 1000 . We further show that each individual resonance wavelength can be independently tuned over a $50 \%$ spectral range by modifying a single geometrical antenna parameter, which provides flexible control over the collective spectral response. Enabled by the broad spectral coverage of our design, we introduce a novel sensing method that simultaneously performs chemically specific midinfrared detection and near-infrared refractometry, and demonstrate it experimentally using polymer analyte molecules. The flexibility, uniformly high excitation efficiency, and broad spectral range of our multiresonant self-similar arrays can open up exciting applications in fields such as nanoantenna-enhanced photodetection, photovoltaics, and surfaceenhanced biochemical sensing.

\section{RESULTS AND DISCUSSION}

Self-Similar Multiresonant Array. The concept of the self-similar multiscale array is presented in Figure 1. A periodic subarray of plasmonic dipole antennas with length $L_{1}$, width $W_{1}$, and periodicity $P_{1}$ generates the lowest resonance frequency of the array $\left(f_{1}\right)$. A second subarray of dipoles with all dimensions scaled by a factor of $1 / 2\left(L_{2}=L_{1} / 2, W_{2}=\right.$

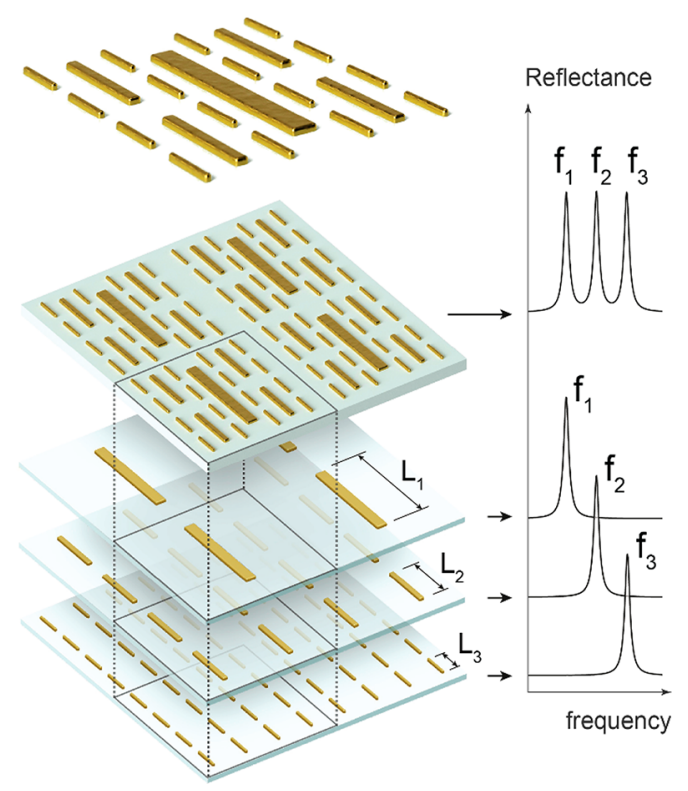

Figure 1. Conceptual view of the multiresonant array. Multiple dipole arrays with different lengths and periodicities are combined to provide multiple IR resonances. The illustration shows the arrangement for a 3rd order fractal array supporting three optical resonances.

$\left.W_{1} / 2, P_{2}=P_{1} / 2\right)$ provides a resonance at two-times the initial resonance frequency $\left(f_{2}=2 f_{1}\right)$. The two subarrays are combined to form a multiresonant array by maximizing the distance between the elements. The factor of 2 scaling provides perfect matching between the different periodicities and avoids overlap between the nanoantennas. A third resonance is added to the array by incorporating a subarray scaled by a factor of 1 / 4 , resonating at $4 \times$ the initial frequency $\left(f_{3}=4 f_{1}\right)$. The full multiresonant structure is created by repeating this fractal-like generation procedure, resulting in a multiscale geometry with self-similar characteristics and with as many resonances as fractal iterations.

The proposed technique is applied to design a multiresonant array covering a broad spectral range from the near-infrared to the mid-infrared region of the spectrum. This range is crucial for vibrational sensing and the proposed multiresonant technique is of particular interest in infrared biosensors, which require simultaneous enhancement of multiple vibrational bands for analyzing complex samples containing various analytes such as lipids, proteins, or other biological components. $^{26,41}$ The dimensions of the dipoles are optimized to provide a first resonance $\left(f_{1}\right)$ at a $10 \mu \mathrm{m}$ wavelength $(1000$ $\left.\mathrm{cm}^{-1}\right)$, with higher order resonances located at shorter wavelengths uniformly spaced in octaves. Scanning electron microscope (SEM) and atomic force microscope (AFM) images of the 3-peak multiresonant arrays are shown in Figure $2 a$ and $b$, respectively. SEM images of the multiresonant array unit cells for the different orders $(N=1,2,3,4)$ of the fractal structure are shown in Figure $2 c$, and their corresponding reflectance spectra are shown in Figure $2 \mathrm{~d}$. The measured spectra clearly show that the number of resonances is equal to the order of the fractal structure. In all cases, the measured frequency of the fundamental resonance is approximately at $1000 \mathrm{~cm}^{-1}$, and the higher order resonances have a frequency scaling factor equal to two. This multiresonant behavior is observed only when the polarization of the infrared light is aligned with the dipolar axis, while the 


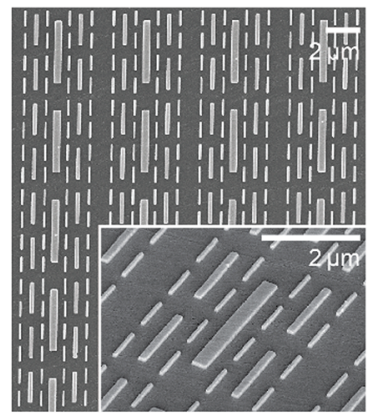

b

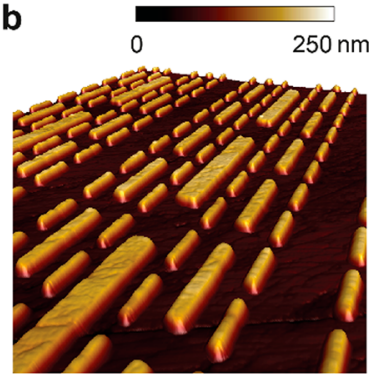

C
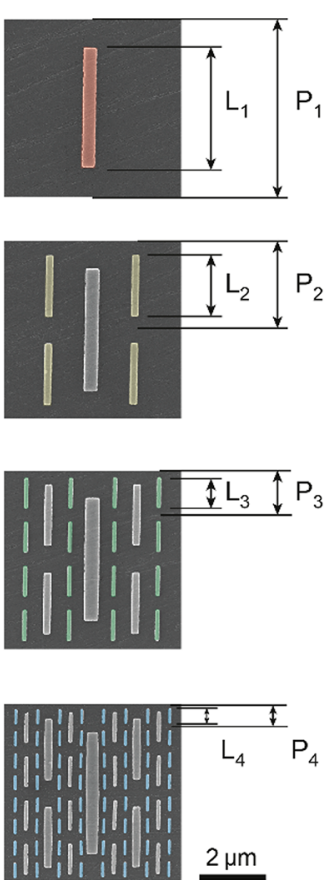

d Reflectance (\%)

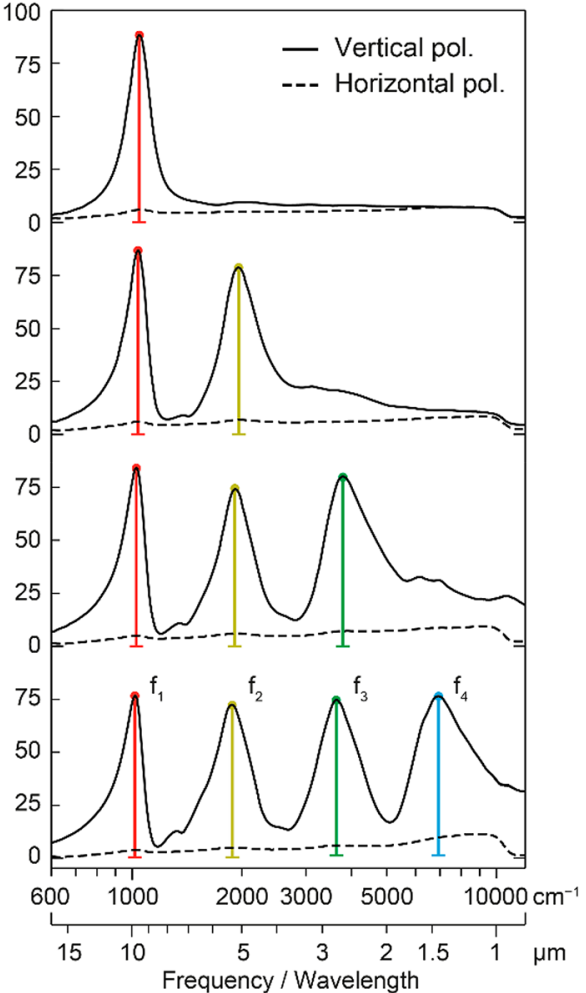

Figure 2. (a) Scanning electron and (b) atomic force microscopy images of the 3-peak multiresonant array. (c) Scanning electron microscope images and (d) infrared reflectance spectra of the multiresonant arrays with 1, 2, 3 and 4 resonance peaks. Solid (dashed) curves correspond to the excitation with vertical (horizontal) incident light polarization. Each additional resonance is supported by a new dipole subarray with shorter length and periodicity (from top to bottom). The nominal dimensions of the array are periodicity $P_{1}=5.20, P_{2}=2.60, P_{3}=1.30, P_{4}=0.65$, dipole length $L_{1}=3.60, L_{2}=1.80, L_{3}=0.90, L_{4}=0.45$, dipole width $W_{1}=0.40, W_{2}=0.20, W_{3}=0.10, W_{4}=0.07$ and thickness $t=0.10$ (units in $\mu \mathrm{m}$ ).

array becomes essentially transparent for the transversal polarization.

A logarithmic frequency axis is used in Figure $2 \mathrm{~d}$ to capture the multiscale log-periodic properties of the self-similar array. ${ }^{42}$ The spectral response at the higher order modes in our multiscale array is a frequency-scaled copy of the fundamental mode, which corresponds to a horizontal shift in a logarithmic axis. Additionally, the uniform scaling factor results in an even distribution of the resonance peaks over the logarithmic frequency axis.

It is remarkable that, despite the densely packed structure of the self-similar array, especially for $N=3$ and 4, all resonance peaks are well-defined and reach high reflectance intensities (>70\%), indicating that the electromagnetic near-field coupling between different subarrays is relatively low. Furthermore, it is important to emphasize the strikingly broad wavelength range covered by the multiresonant array, reaching almost a 1:10 resonance frequency ratio. In particular, the 4-peak array is able to operate from the long-wavelength infrared $(10 \mu \mathrm{m})$ to the near-infrared telecom wavelengths $(1.55 \mu \mathrm{m})$. In general, the maximum number of resonances $N_{\max }$ for a given fractal design is determined by the requirement of geometrically nonoverlapping dipoles for all subarrays. The critical minimum gap distance $D_{\text {min }}$ is located between the dipoles of first and $N^{\text {th }}$ order and can be calculated from the unit cell parameters via $D_{\text {min }}=\left(P_{N}-W_{1}-W_{N}\right) / 2$, where $P_{N}=P_{1} / 2^{N-1}$ is the periodicity of the $N^{\text {th }}$ subarray and $W_{1}$ and $W_{N}$ are the respective dipole widths. In the case of $P_{1}=5.20 \mu \mathrm{m}$ and $W_{1}=$ $W_{N}=0.1 \mu \mathrm{m}$, this results in a maximum of five resonances with a minimum gap size of $D_{\text {min }}=62 \mathrm{~nm}$, which is still sufficiently large to avoid strong near-field coupling between the adjacent dipole elements. ${ }^{41}$ If needed, the number of resonances can be increased further by tailoring both the array periodicity and the width of the individual dipoles.

Numerical Analysis and Optical fields. The electromagnetic response and the underlying physical mechanisms in the self-similar structure are analyzed using numerical simulations. In the following, the third-order fractal array is considered, which captures the main properties of the multiscale structure. The electromagnetic response of the 3peak multiresonant array is simulated using a full-wave frequency-domain solver of Maxwell's equations. The calculated spectrum is presented in Figure $3 \mathrm{a}$ and is in good agreement with the experimental results. In particular, it accurately reproduces the spectral positions, lineshapes and line widths of the resonances. In Figure $3 b$, we plot the simulated near-field intensity of the structure for each resonance frequency. It is clear that only the corresponding set of dipoles is active at each resonance frequency and a low coupling level is observed between different subarrays. The maximum near-field enhancement takes place at the tips of the corresponding dipoles, reaching peak intensity enhancements between three and four orders of magnitude. Most significantly, the field enhancement at each resonance of the multiresonant array is almost identical to that of the singleresonance structure obtained by isolating the corresponding subarray (Figure 3c). 


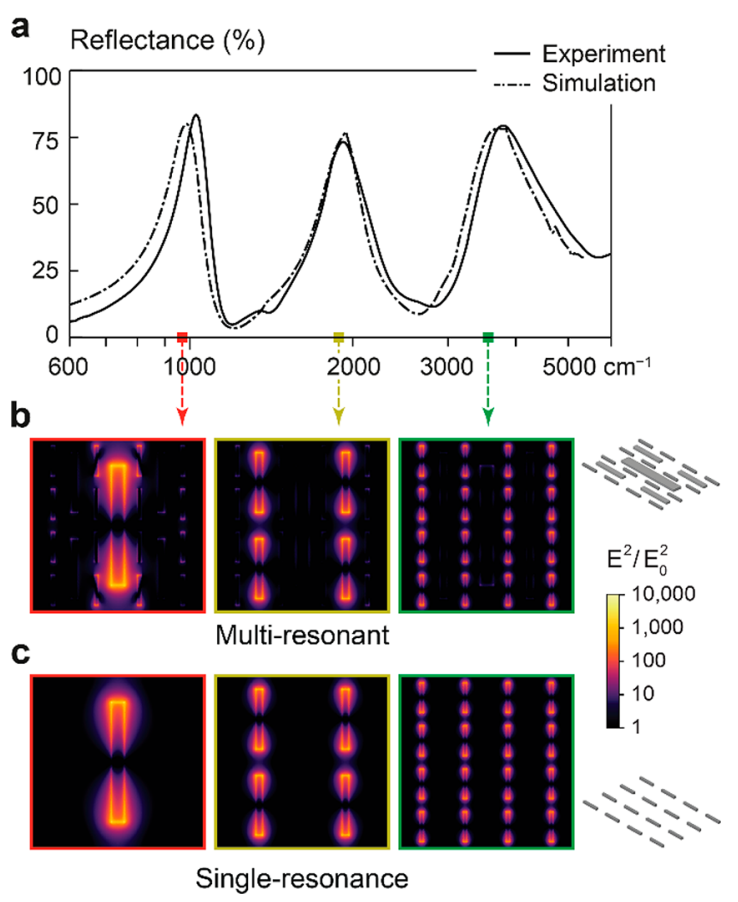

Figure 3. (a) Comparison between electromagnetic simulations and measurements of the 3-peak multiresonant array. (b) Simulated nearfield intensity distributions of the multiresonant array for each of the three resonance frequencies. Only the corresponding subarray is electromagnetically excited for each frequency. (c) Simulated nearfield intensity distribution of the corresponding single-frequency arrays. The field enhancements of the multiresonant array are almost identical to those of the isolated single-resonance subarrays.

Spectral Tunability. The allocation of the different resonance modes on separate nanostructure subarrays provides an additional advantage: each resonance can be individually adjusted by scaling the geometry of the corresponding subarray. In Figure 4a, we present the reflectance spectra of the multiresonant array when the length of each dipole set $\left(L_{1}\right.$, $\left.L_{2}, L_{3}\right)$ is individually scaled in increments of $8 \%$. The spectral data show that the resonance corresponding to the scaled dipole subarray is shifted, while the other resonance peaks remain virtually unaffected. This result contrasts with traditional multiband arrays composed of individual multiresonant nanoantenna elements, where any geometrical modification simultaneously affects all the resonance modes. The mutual independence of the modes of our multiresonant array allows its response to be easily tailored to provide strong field enhancements for specific target frequencies.

Significantly, the spectral position of each resonance can be varied over a wide wavelength range. The spectral tuning range measured from the shortest to the longest resonance wavelength is $47 \%, 57 \%$, and $60 \%$ for the first, second, and third resonance modes, respectively. For comparison, Figure $4 \mathrm{~b}$ presents the spectra of single-resonance arrays with dimensions identical to the corresponding subarrays in the multiscale structure. Interestingly, the resonance frequencies of the multiresonant arrays match almost perfectly those of the single-resonance arrays. This is a clear indication of low nearfield coupling between subarrays, which otherwise would have produced frequency shifts associated with the bonding and antibonding modes. The achieved spectral tuning ranges in the multiresonant structures are almost identical to those in their single-resonance counterparts. The tuning range is currently limited by the excitation of the grating modes on the highfrequency side and by the antenna overlap $\left(L_{\mathrm{i}}>P_{\mathrm{i}}\right)$ on the lowfrequency side of the spectrum.

TCMT Modeling. The electromagnetic response of the multiresonant array can be understood with temporal coupledmode theory (TCMT), ${ }^{43}$ allowing for the straightforward extension of our multiresonant concept to other target wavelengths. TCMT models the optical platform as a set of $N$ resonance modes with amplitudes $\mathbf{a}=\left(a_{1}, \ldots, a_{N}\right)$, which are coupled both mutually and to external ports. The timeevolution of the modes' amplitudes (a) and the incident $\left(s_{+}\right)$ and scattered waves $\left(s_{-}\right)$are determined by the following expressions:

$$
\begin{aligned}
& \frac{\mathrm{d}}{\mathrm{d} t} \mathbf{a}=(j \Omega-\Gamma) \mathbf{a}+K^{T} s_{+} \\
& s_{-}=C s_{+}+D \mathbf{a}
\end{aligned}
$$

where $\Omega$ is a $N \times N$ matrix determined by the mode resonance frequencies $\left(\omega_{01}, \ldots, \omega_{0 \mathrm{~N}}\right)$ and the near-field coupling rates; $\Gamma$ is determined by the mode radiative damping $\left(\gamma_{1}, \ldots, \gamma_{\mathrm{N}}\right)$ and radiative coupling rates; $D$ corresponds to port-mode coupling; and $C$ is the direct port-port coupling. Taking into account the even symmetry of the modes with respect to the two ports, the rest of the matrices are calculated as follows: ${ }^{44}$

$$
\begin{aligned}
& \Gamma=\left(\sqrt{\gamma_{1}} \sqrt{\gamma_{2}} \cdots \sqrt{\gamma_{\mathrm{N}}}\right)^{\mathrm{T}}\left(\sqrt{\gamma_{1}} \sqrt{\gamma_{2}} \cdots \sqrt{\gamma_{\mathrm{N}}}\right) \\
& K=D=\left(\begin{array}{ll}
1 & 1
\end{array}\right)^{\mathrm{T}}\left(\sqrt{\gamma_{1}} \sqrt{\gamma_{2}} \cdots \sqrt{\gamma_{\mathrm{N}}}\right) \\
& C=\left(\begin{array}{rr}
0 & -1 \\
-1 & 0
\end{array}\right)
\end{aligned}
$$

The scattering matrix of the structure, composed of reflection $\left(s_{11}, s_{22}\right)$ and transmission $\left(s_{12}, s_{21}\right)$ coefficients can then be calculated as

$$
s_{-}=\left(\begin{array}{ll}
s_{11} & s_{12} \\
s_{21} & s_{22}
\end{array}\right) s_{+}=\left[C+D(j \omega I-j \Omega+\Gamma)^{-1} K^{\mathrm{T}}\right] s_{+}
$$

where the coupling and damping matrices are determined from eq 3 using the mode resonance frequencies $\left(\omega_{0 i}\right)$ and the mode damping rates $\left(\gamma_{i}\right)$ obtained from single-resonance measurements (Figure S1). The only free parameters in the TCMT model are the near-field coupling rates $\left(\Omega_{i j}, i \neq j\right)$.

In Figure 5a, we compare the experimental reflectance spectrum of the 3-peak multiresonant array and the one obtained from the TCMT model for different values of nearfield coupling $\left(\Omega_{i j}\right)$. The reflectance of single-resonance arrays is represented by shaded areas. The TCMT model accurately captures the main spectral features of the multiresonant reflectance spectrum. Particularly interesting is the asymmetric Fano-type line shape of the lowest frequency peak, which is a result of the spectral overlap between the sharper first-order mode and the second-order mode with broader line width. ${ }^{45}$ Importantly, this asymmetric line shape also emerges when only far-field coupling is considered $\left(\Omega_{i j}=0\right)$. Increasing the near-field coupling in the TCMT model produces a stronger asymmetry of the lowest frequency peak corresponding to the first-order mode and, at the same time, increases the spectral separation between this mode and the one at the high frequency side of the spectrum due to the presence of coupling-induced bonding and antibonding modes. ${ }^{46}$ From the spectral shift of the lowest frequency mode measured in the 

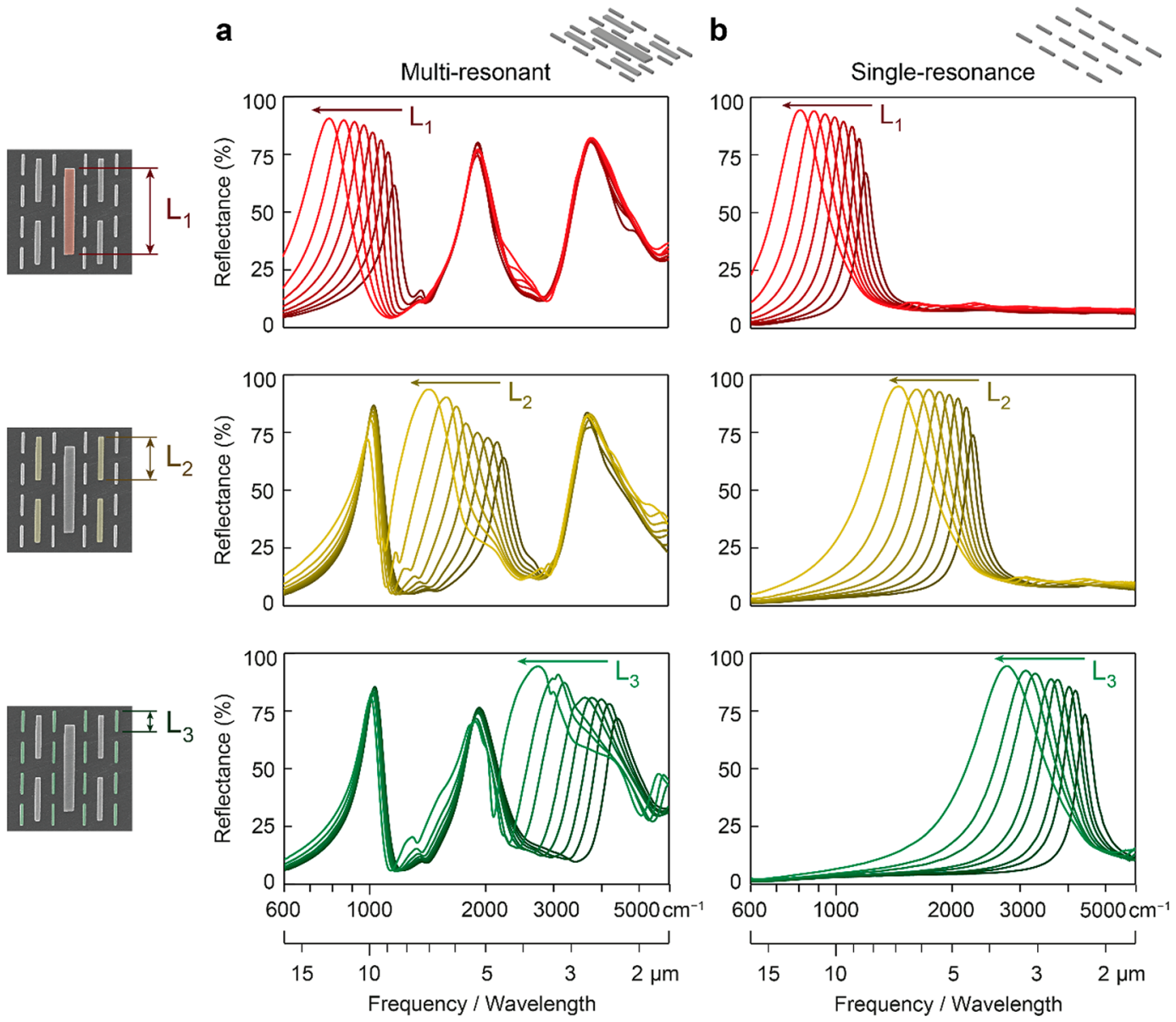

Figure 4. (a) Experimental demonstration of individual resonance tuning in the multiresonant array by adjusting the length of the corresponding subarray dipole. The dipole lengths $L_{1}, L_{2}$ and $L_{3}$ are individually varied in increments of $8 \%\left(L_{1}=2.86-4.90 \mu \mathrm{m}, L_{2}=1.43-2.45 \mu \mathrm{m}, L_{3}=0.71-\right.$ $1.22 \mu \mathrm{m})$ and the periodicities are unmodified $\left(P_{1}=5.20, P_{2}=2.60, P_{3}=1.30 \mu \mathrm{m}\right)$. The 1st resonance shifts from $\lambda_{1}=8.57$ to $12.61 \mu \mathrm{m}$, the $2 \mathrm{nd}$ resonance shifts from $\lambda_{2}=4.44$ to $6.98 \mu \mathrm{m}$, the 3rd resonance shifts from $\lambda_{3}=2.27$ to $3.64 \mu \mathrm{m}$. (b) Experimental reflectance spectra of the isolated single-resonance subarrays composed of dipoles with identical length $\left(L_{\mathrm{i}}\right)$ and periodicity $\left(P_{\mathrm{i}}\right)$ to those in the corresponding multiresonant structure.

experiments, we estimate a coupling rate of $\Omega_{i j}=0.05 \sqrt{\omega_{i} \omega_{j}}(i$ $\neq j)$. This low level of near-field coupling is in agreement with the levels observed in the near-field simulations (Figure 3).

The TCMT model is also able to reproduce the experimental results for different number of modes $(N=1$, 2, 3, and 4), as shown in Figure 5b. Similar to the observations in Figure 5a, each line shape is initially symmetric and becomes progressively asymmetric as the higher order modes are incorporated into the structure. Since the asymmetric line shape is a consequence of the spectral overlap, it depends strongly on the line widths and the spectral separation between the modes. This is clearly observed in Figure 5c, where the length of the second-order dipoles $\left(L_{2}\right)$ is changed from 1.54 $\mu \mathrm{m}$ (blue) to $2.45 \mu \mathrm{m}$ (red) in incremental steps of $8 \%$. The change in the antenna length corresponds to a $50 \%$ resonance tuning in the 1450 to $2130 \mathrm{~cm}^{-1}$ (4.7 to $6.9 \mu \mathrm{m}$ ) spectral range. Longer second-order dipoles (red curves) generate broader resonances at lower frequencies, producing higher spectral overlap with the first-order resonance. The result is a clear increase in the asymmetry of the first-order mode, which is well captured by the TCMT model.

Simultaneous Operation of Multiband SEIRA and Refractometry. Due to the broad spectral coverage of its constituent resonances, our multiresonant plasmonic design is ideally suited for a combined sensing scheme that simultaneously provides two of the main label-free detection mechanisms: surface-enhanced IR spectroscopy and refractometry.

To illustrate the power of this approach, we implement our technique for the chemically specific detection of the two polymers poly(methyl methacrylate) (PMMA) and poly(styrene- $b$-1,4-butadiene- $b$-styrene) (PS-PBd-PS). At mid-IR frequencies, both polymers exhibit distinct absorption fingerprints over a wide spectral range (Figure 6a, top). Importantly, we can easily tailor the optical response of our design through the tuning of the dipole lengths $L_{1}, L_{2}$, and $L_{3}$ so that each of the three resonance peaks (\#1, \#2, and \#3) overlaps with a target vibrational band of interest. In contrast, the highest frequency resonance peak (\#4) is designed to be in the nearIR, which is free of vibrational modes and well suited for refractometric detection.

The reflectance spectrum of the fabricated multiresonant antenna array clearly shows these tailored resonance properties as well as the analyte-induced modulation of the resonance lineshapes after deposition of the polymer layers (Figure 6a, bottom). In particular, peak $\# 1$ is tuned to detect a characteristic mode of PS-PBd-PS at $966 \mathrm{~cm}^{-1}$, which corresponds to the trans-alkene out of plane bending mode 
a
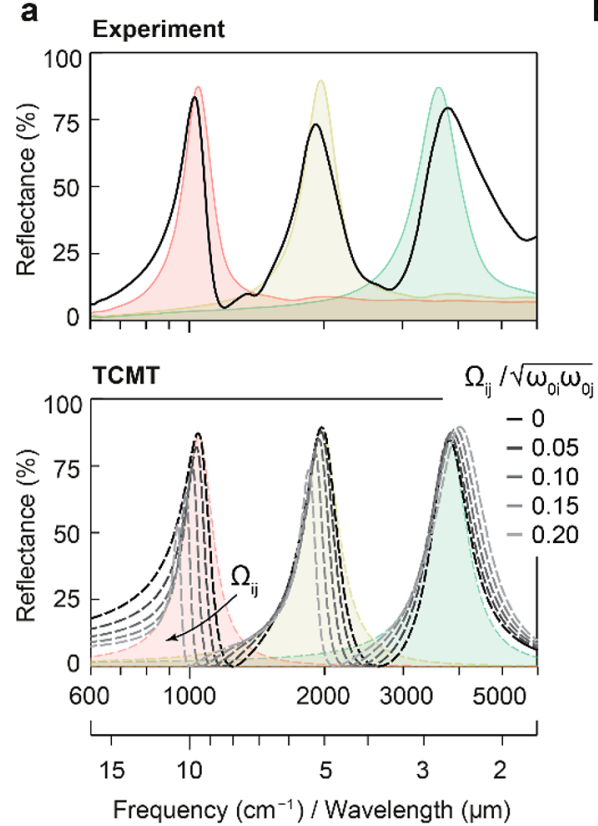

b

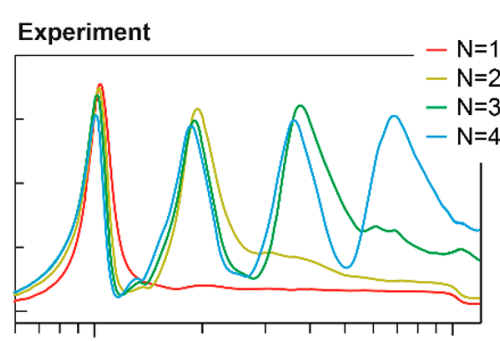

TСMT

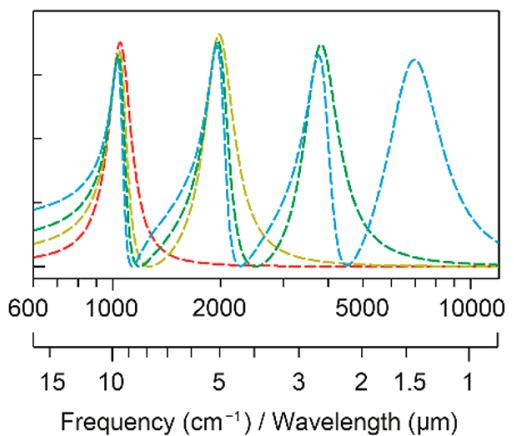

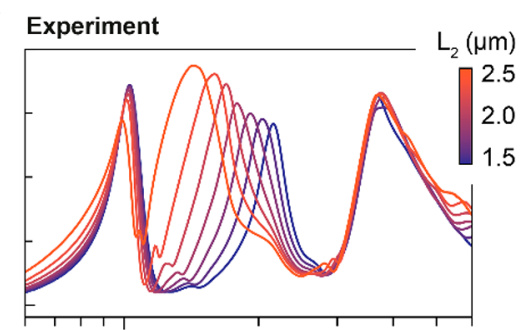

TCMT

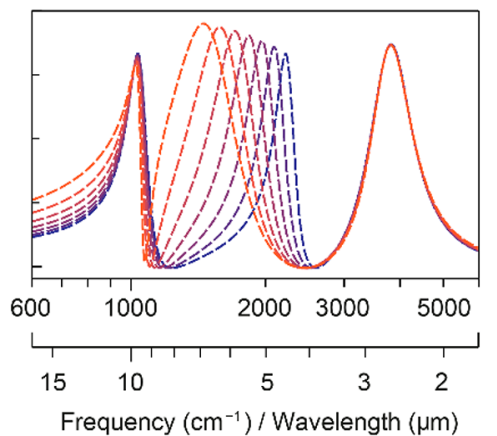

Figure 5. Temporal coupled-mode theory (TMCT) modeling. (a) Experimental (top) and TCMT calculated (bottom) spectra of the 3-peak multiresonant array for different values of near-field coupling $\left(\Omega_{i j} / \sqrt{\omega_{0 i} \omega_{0 j}}, i \neq j\right)$. Shaded areas represent the corresponding single-resonance spectra. (b) Experimental and TCMT calculated spectra of multiresonant arrays with different number of resonances: 1-peak (red), 2-peak (yellow), 3-peak (green), and 4-peak (blue). (c) Experimental and TCMT calculated spectra of the 3-peak multiresonant array for different lengths of the 2nd-order dipoles $\left(L_{2}\right)$.

of the butadiene moieties, ${ }^{47,48}$ peak $\# 2$ detects the $\mathrm{C}=\mathrm{O}$ stretching mode of $\mathrm{PMMA}^{49}$ at $1736 \mathrm{~cm}^{-1}$, and peak \#3 provides spectral overlap with the various $\mathrm{C}-\mathrm{H}$ modes of the two polymers. ${ }^{50}$ For PMMA, the main $\mathrm{C}-\mathrm{H}$ modes are found at 2840, 2950, and $2996 \mathrm{~cm}^{-1}$, whereas for PS-PBs-PS, they are observed at 2846, 2920, and $3006 \mathrm{~cm}^{-1}$. To further highlight the SEIRA performance, Figure $6 \mathrm{~b}$ shows the measured reflectance spectra when the dipole lengths $L_{1}, L_{2}$, and $L_{3}$ are adjusted around their optimized values. The visibility of the target vibrational bands, which show up as reflectance dips, increases as the resonance frequency of the corresponding antenna elements is tuned toward the spectral location of the absorption band, highlighting the importance of precisely controlling the spectral positions of multiple resonances in a single optical sensor design. The wide spectral operating range of our design also illustrates the intrinsic scaling law of the SEIRA enhancement, which has recently been shown to be inversely proportional to the third power of the resonance frequency $f$ via $(1 / f)^{3}$. $^{51}$ In accordance with this scaling behavior, we observe reduced absorption band visibility for the high frequency peak \#3 compared to the low frequency peak $\# 1$, even though both peaks have similar extinction amplitude and the absorption strengths of the sensed bands are comparable.

When comparing the reflectance spectrum of the multiresonant sensor before and after deposition of the polymers, a red-shift of the resonance position can be observed for all peaks. To better quantify this behavior, we extract the relative frequency shift for all four resonance peaks (Figure 6c). We find that the spectral shift is largest for the highest frequency peak, which corresponds to the smallest antenna dipole with length $L_{4}$. This is due to the stronger confinement of the electric near-fields for higher frequencies, which produces a stronger overlap with the thin molecular layer and therefore leads to a better refractometric sensitivity. In addition, resolving the spectral shifts in the near-IR range is facilitated by the absence of interfering molecular vibrational signals. With this dual sensing scheme, we are able to take advantage of both chemical identification and refractometric detection simultaneously, opening up new opportunities for the interrogation of nanometric analyte layers. Our multiresonant design is capable of leveraging additional degrees of freedom to obtain further flexibility, such as (1) different geometrical scaling factors, (2) different types of lattices, and (3) different types of nanoantenna geometries. Specifically, it is not limited to the previously described ratio of 1:2 for the dipole array periodicities, but can also be applied for other periodicity ratios such as $1: 1.5$ or $1: 3$. Furthermore, our design can easily be extended to utilize more complex lattice types such as rhombic or hexagonal, opening up a new design space for multiresonant geometries (Figure S2).

\section{CONCLUSIONS}

We presented a method for producing multiresonant structures able to enhance optical near-fields by more than three orders of magnitude for frequencies covering an ultrawide spectral range from the long-wavelength IR $(10 \mu \mathrm{m})$ to the near-IR $(1.5 \mu \mathrm{m})$. The structure consisting of self-similar subarrays supports welldefined resonance peaks and, most importantly, all of them are efficiently excited with nearly equal amplitudes. We controlled each of the resonances over a $50 \%$ frequency tuning range while preserving the integrity of the other resonances thanks to the low near-field coupling between the subarrays. We used temporal coupled-mode theory to explain the electromagnetic response of our multiscale arrays and demonstrated an intuitive scaling scheme that allows to tailor the resonances at specific spectral bands. Strikingly, we leveraged the broad spectral coverage of the design to implement a novel biochemical 

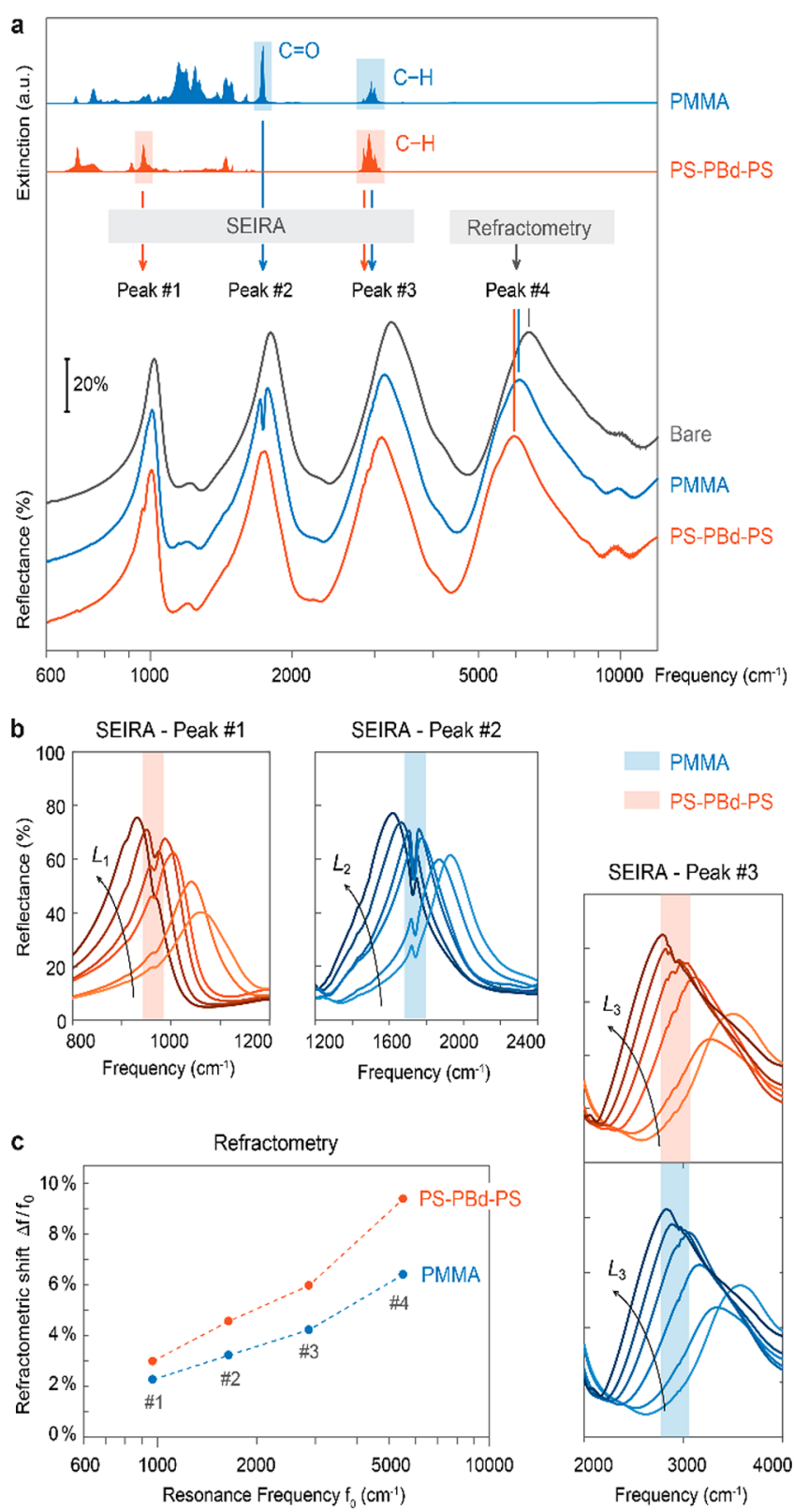

Figure 6. (a) Reflectance spectra of the multiresonant sensor chip before and after coating with either PMMA or PS-PBd-PS analytes. (b) SEIRA performance for the reflectance peaks \#1, \#2, and \#3 when the lengths of the corresponding dipole antennas are varied around the optimum value. Visibility of the target vibrational modes is best when the antenna resonance frequency overlaps with the spectral location of the absorption band. (c) Refractometric resonance shift is strongest for peak \#4 with the highest resonance frequency located in the near-IR.

sensing technique, which simultaneously performs surfaceenhanced mid-IR absorption spectroscopy and near-IR refractometry. The near-IR resonances are more sensitive to refractive index changes due to higher field confinements, while the multiple mid-IR resonances enable chemically specific detection. The performance and versatility of our technique makes it a powerful tool for multiband optical devices with particular interest for infrared sensors.

\section{MATERIALS AND METHODS}

Nanofabrication. Infrared nanoantennas are fabricated on $\mathrm{CaF}_{2}$ substrates using a lift-off process. Poly(methyl methacrylate) (PMMA) is used as the pattern defining electron beam resist and a lower molecular weight sublayer is used to aid lift-off. A $5 \mathrm{~nm}$ thick gold layer is sputtered before the exposure to minimize charging effects and is removed immediately after exposure by wet etching in $\mathrm{KI}+\mathrm{I}_{2}$ solution. The resist is developed in 1:3 MiBK/IPA solution for $1 \mathrm{~min}$ and rinsed in IPA. A $100 \mathrm{~nm}$ thick layer of gold is evaporated using $5 \mathrm{~nm}$ of chromium as an adhesion layer. The lift-off is carried out in acetone and resist residue is eliminated by oxygen plasma.

Electromagnetic Simulations. The reflectance of the nanoantenna arrays and their optical near-fields are simulated using frequency domain finite element Maxwell solver on a tetrahedral mesh contained in CST Studio Suite 2016. The periodic structure is modeled by enforcing periodic boundary conditions surrounding the unit cell and exciting the structure with the corresponding Floquet modes. The number of modes is chosen so that all nonevanescent Floquet modes are considered in the simulation. The permittivity of gold has been taken from Palik ${ }^{52}$ and a refractive index $n=1.4$ is used for the $\mathrm{CaF}_{2}$ substrate. The simulation mesh is iteratively refined until the simulation results fulfill the convergence criteria.

Optical Measurements. The reflectance spectra of the nanoantenna arrays are measured with a Fourier transform infrared (FT-IR) spectrometer (Bruker Vertex 80v) coupled to an infrared microscope (Bruker Hyperion 3000). A $15 \times(\mathrm{NA}=$ 0.4) Cassegrain objective in combination with an infrared polarizer and a knife-edge aperture is used to illuminate and collect the reflected light from the arrays. Two different configurations of the FT-IR are used to cover the full spectral range of operation of the multiresonant arrays. The midinfrared region of the spectrum is measured using a Globar source and a $\mathrm{KBr}$ beamsplitter. The near-IR spectrum is measured using a tungsten lamp and $\mathrm{CaF}_{2}$ beamsplitter. In both cases, a liquid-nitrogen cooled mercury-cadmiumtellurium detector is used. The measured mid- and near-IR spectra are matched to produce the broadband spectra.

Analyte Preparation. For the chemical sensing experiments, poly (methyl methacrylate) (PMMA) and poly(styrene$b$-1,4-butadiene- $b$-styrene) (PS-PBd-PS) are diluted in methoxybenzene (i.e., anisole) and chloroform, respectively. Thin polymer layers are created on the multiresonant antenna chip by spin coating these dilutions at $6000 \mathrm{rpm}$ for $60 \mathrm{~s}$. The same multiresonant chip is used for both measurements by employing a thorough solvent-based cleaning step before coating of the individual polymer layers.

\section{ASSOCIATED CONTENT}

\section{Supporting Information}

The Supporting Information is available free of charge on the ACS Publications website at DOI: 10.1021/acsphotonics.8b01050.

Comparison between experimental spectra and Lorentzian models for single-resonance arrays and extracted TCMT parameters (resonance frequencies, intrinsic, and radiative damping rates) in Figure S1. Extension of the multiresonant array to different periodicity ratios, 
oblique lattices and other lattice types in Figure S2 (PDF).

\section{AUTHOR INFORMATION}

\section{Corresponding Author}

*E-mail: hatice.altug@epfl.ch.

\section{ORCID}

Andreas Tittl: 0000-0003-3191-7164

Aurelian John-Herpin: 0000-0002-3554-1574

Hatice Altug: 0000-0001-5522-1343

\section{Author Contributions}

${ }^{\S}$ These authors contributed equally.

\section{Notes}

The authors declare no competing financial interest.

\section{ACKNOWLEDGMENTS}

We thank M. Soler and D. Etezadi for help with polymer preparation. The research leading to these results has received funding from the European Union Seventh Framework Programme under grant agreement no. 625673 GRYPHON and no. 609416 ICFONest+, from the European Union's Horizon 2020 Framework Programme for Research and Innovation under grant agreement no.665667, and from European Research Council (ERC) under Grant Agreement No. 682167 VIBRANT-BIO. We acknowledge financial support from the Swiss National Science Foundation through Project No. 133583. We also acknowledge École Polytechnique Fédérale de Lausanne and Center of MicroNano Technology for nanofabrication.

\section{REFERENCES}

(1) Mühlschlegel, P.; Eisler, H. J.; Martin, O.; Hecht, B.; Pohl, D. Resonant Optical Antennas. Science 2005, 308 (10), 1607-1609.

(2) Schuck, P. J.; Fromm, D. P.; Sundaramurthy, A.; Kino, G. S.; Moerner, W. E. Improving the Mismatch between Light and Nanoscale Objects with Gold Bowtie Nanoantennas. Phys. Rev. Lett. 2005, 94 (1), 017402.

(3) Alù, A.; Engheta, N. Input Impedance, Nanocircuit Loading, and Radiation Tuning of Optical Nanoantennas. Phys. Rev. Lett. 2008, 101 (4), 043901.

(4) Novotny, L.; van Hulst, N. Antennas for Light. Nat. Photonics 2011, 5 (2), 83-90.

(5) Anker, J. N.; Hall, W. P.; Lyandres, O.; Shah, N. C.; Zhao, J.; Van Duyne, R. P. Biosensing with Plasmonic Nanosensors. Nat. Mater. 2008, 7, 442-453.

(6) Yanik, A. A.; Huang, M.; Kamohara, O.; Artar, A.; Geisbert, T. W.; Connor, J. H.; Altug, H. An Optofluidic Nanoplasmonic Biosensor for Direct Detection of Live Viruses from Biological Media. Nano Lett. 2010, 10 (12), 4962-4969.

(7) Tittl, A.; Yin, X.; Giessen, H.; Tian, X.-D.; Tian, Z.-Q.; Kremers, C.; Chigrin, D. N.; Liu, N. Plasmonic Smart Dust for Probing Local Chemical Reactions. Nano Lett. 2013, 13 (4), 1816-1821.

(8) Adato, R.; Yanik, A. A.; Amsden, J. J.; Kaplan, D. L.; Omenetto, F. G.; Hong, M. K.; Erramilli, S.; Altug, H. Ultra-Sensitive Vibrational Spectroscopy of Protein Monolayers with Plasmonic Nanoantenna Arrays. Proc. Natl. Acad. Sci. U. S. A. 2009, 106 (46), 19227-19232.

(9) Etezadi, D.; Warner, J. B.; Lashuel, H. A.; Altug, H. ACS Sens. 2018, 3 (6), 1109-1117.

(10) John-Herpin, A.; Tittl, A.; Altug, H. ACS Photonics 2018, 5 (10), 4117-4124.

(11) Yu, Z.; Raman, A.; Fan, S. Fundamental Limit of Nanophotonic Light Trapping in Solar Cells. Proc. Natl. Acad. Sci. U. S. A. 2010, 107 (41), 17491-17496.
(12) Atwater, H. A.; Polman, A. Plasmonics for Improved Photovoltaic Devices. Nat. Mater. 2010, 9 (3), 205-213.

(13) Aieta, F.; Kats, M. A.; Genevet, P.; Capasso, F. Multiwavelength Achromatic Metasurfaces by Dispersive Phase Compensation. Science 2015, 347 (6228), 1342-1345.

(14) Awazu, K.; Fujimaki, M.; Rockstuhl, C.; Tominaga, J.; Murakami, H.; Ohki, Y.; Yoshida, N.; Watanabe, T. A Plasmonic Photocatalyst Consisting of Silver Nanoparticles Embedded in Titanium Dioxide. J. Am. Chem. Soc. 2008, 130 (5), 1676-1680.

(15) Knight, M. W.; Sobhani, H.; Nordlander, P.; Halas, N. J. Photodetection with Active Optical Antennas. Science 2011, 332 (6030), 702-704.

(16) Zuloaga, J.; Nordlander, P. On the Energy Shift between NearField and Far-Field Peak Intensities in Localized Plasmon Systems. Nano Lett. 2011, 11 (3), 1280-1283.

(17) D’Andrea, C.; Bochterle, J.; Toma, A.; Huck, C.; Neubrech, F.; Messina, E.; Fazio, B.; Maragò, O. M.; Di Fabrizio, E.; Lamy de La Chapelle, M.; et al. Optical Nanoantennas for Multiband SurfaceEnhanced Infrared and Raman Spectroscopy. ACS Nano 2013, 7 (4), 3522-3531.

(18) Le, F.; Brandl, D. W.; Urzhumov, Y. A.; Wang, H.; Kundu, J.; Halas, N. J.; Aizpurua, J.; Nordlander, P. Metallic Nanoparticle Arrays: A Common Substrate for Both Surface-Enhanced Raman Scattering and Surface-Enhanced Infrared Absorption. ACS Nano 2008, 2 (4), 707-718.

(19) Neubrech, F.; Pucci, A.; Cornelius, T. W.; Karim, S.; GarcíaEtxarri, A.; Aizpurua, J. Resonant Plasmonic and Vibrational Coupling in a Tailored Nanoantenna for Infrared Detection. Phys. Rev. Lett. 2008, 101 (15), 157403.

(20) Adato, R.; Altug, H. In-Situ Ultra-Sensitive Infrared Absorption Spectroscopy of Biomolecule Interactions in Real Time with Plasmonic Nanoantennas. Nat. Commun. 2013, 4 (1), 2154.

(21) Aslan, E.; Aslan, E.; Wang, R.; Hong, M. K.; Erramilli, S.; Turkmen, M.; Saracoglu, O. G.; Dal Negro, L. Multispectral CesaroType Fractal Plasmonic Nanoantennas. ACS Photonics 2016, 3 (11), $2102-2111$

(22) Hasan, D.; Ho, C. P.; Pitchappa, P.; Yang, B.; Yang, C.; Lee, C. Thermoplasmonic Study of a Triple Band Optical Nanoantenna Strongly Coupled to Mid IR Molecular Mode. Sci. Rep. 2016, 6 (1), 22227.

(23) Gottheim, S.; Zhang, H.; Govorov, A. O.; Halas, N. J. Fractal Nanoparticle Plasmonics: The Cayley Tree. ACS Nano 2015, 9 (3), 3284-3292.

(24) Volpe, G.; Volpe, G.; Quidant, R. Fractal Plasmonics: Subdiffraction Focusing and Broadband Spectral Response by a Sierpinski Nanocarpet. Opt. Express 2011, 19 (4), 3612.

(25) Wallace, G. Q.; Foy, H. C.; Rosendahl, S. M.; LagugnéLabarthet, F. Dendritic Plasmonics for Mid-Infrared Spectroscopy. J. Phys. Chem. C 2017, 121 (17), 9497-9507.

(26) Chen, K.; Adato, R.; Altug, H. Dual-Band Perfect Absorber for Multispectral Plasmon-Enhanced Infrared Spectroscopy. ACS Nano 2012, 6 (9), 7998-8006.

(27) Jiang, Z. H.; Yun, S.; Toor, F.; Werner, D. H.; Mayer, T. S. Conformal Dual-Band Near-Perfectly Absorbing Mid-Infrared Metamaterial Coating. ACS Nano 2011, 5 (6), 4641-4647.

(28) Cetin, A. E.; Turkmen, M.; Aksu, S.; Altug, H. NanoparticleBased Metamaterials as Multiband Plasmonic Resonator Antennas. IEEE Trans. Nanotechnol. 2012, 11 (1), 208-212.

(29) Turkmen, M.; Aksu, S.; Çetin, A. E.; Yanik, A. A.; Altug, H. Multi-Resonant Metamaterials Based on UT-Shaped Nano-Aperture Antennas. Opt. Express 2011, 19 (8), 7921.

(30) Wu, Z.; Kelp, G.; Yogeesh, M. N.; Li, W.; McNicholas, K. M.; Briggs, A.; Rajeeva, B. B.; Akinwande, D.; Bank, S. R.; Shvets, G.; et al. Dual-Band Moiré Metasurface Patches for Multifunctional Biomedical Applications. Nanoscale 2016, 8 (43), 18461-18468.

(31) Wallace, G. Q.; Tabatabaei, M.; Hou, R.; Coady, M. J.; Norton, P. R.; Simpson, T. S.; Rosendahl, S. M.; Merlen, A.; LagugnéLabarthet, F. Superimposed Arrays of Nanoprisms for Multispectral Molecular Plasmonics. ACS Photonics 2016, 3 (9), 1723-1732. 
(32) Alaee, R.; Lehr, D.; Filter, R.; Lederer, F.; Kley, E.-B.; Rockstuhl, C.; Tünnermann, A. Scattering Dark States in Multiresonant Concentric Plasmonic Nanorings. ACS Photonics 2015, 2 (8), 1085-1090.

(33) Boriskina, S. V.; Dal Negro, L. Multiple-Wavelength Plasmonic Nanoantennas. Opt. Lett. 2010, 35 (4), 538-540.

(34) Blanchard, R.; Boriskina, S. V.; Genevet, P.; Kats, M. A.; Tetienne, J.-P.; Yu, N.; Scully, M. O.; Dal Negro, L.; Capasso, F. Multi-Wavelength Mid-Infrared Plasmonic Antennas with Single Nanoscale Focal Point. Opt. Express 2011, 19 (22), 22113-22124.

(35) Ünlü, E. S.; Tok, R. U.; Şendur, K. Broadband Plasmonic Nanoantenna with an Adjustable Spectral Response. Opt. Express 2011, 19 (2), 1000-1006.

(36) Tok, R. U.; Şendur, K. Femtosecond Pulse Shaping Using Plasmonic Snowflake Nanoantennas. Phys. Rev. A: At., Mol., Opt. Phys. 2011, 84 (3), 033847.

(37) Aouani, H.; Ś́pová, H.; Rahmani, M.; Navarro-Cia, M.; Hegnerová, K.; Homola, J.; Hong, M.; Maier, S. A. Ultrasensitive Broadband Probing of Molecular Vibrational Modes with Multifrequency Optical Antennas. ACS Nano 2013, 7 (1), 669-675.

(38) Navarro-Cia, M.; Maier, S. A. Broad-Band Near-Infrared Plasmonic Nanoantennas for Higher Harmonic Generation. ACS Nano 2012, 6 (4), 3537-3544.

(39) Hughes, T. W.; Fan, S. Plasmonic Circuit Theory for Multiresonant Light Funneling to a Single Spatial Hot Spot. Nano Lett. 2016, 16 (9), 5764-5769.

(40) Maksymov, I. S.; Davoyan, A. R.; Miroshnichenko, A. E.; Simovski, C.; Belov, P.; Kivshar, Y. S. Multifrequency Tapered Plasmonic Nanoantennas. Opt. Commun. 2012, 285 (5), 821-824.

(41) Rodrigo, D.; Tittl, A.; Ait-Bouziad, N.; John-Herpin, A.; Limaj, O.; Kelly, C.; Yoo, D.; Wittenberg, N. J.; Oh, S.; Lashuel, H. A.; et al. Resolving Molecule-Specific Information in Dynamic Lipid Membrane Processes with Multi-Resonant Infrared Metasurfaces. Nat. Commun. 2018, 9 (1), 2160.

(42) Puente-Baliarda, C.; Romeu, J.; Pous, R.; Cardama, A. On the Behavior of the Sierpinski Multiband Fractal Antenna. IEEE Trans. Antennas Propag. 1998, 46 (4), 517-524.

(43) Haus, H. A. Waves and Fields in Optoelectronics; Prentice Hall, 1983.

(44) Suh, W.; Wang, Z.; Fan, S. Temporal Coupled-Mode Theory and the Presence of Non-Orthogonal Modes in Lossless Multimode Cavities. IEEE J. Quantum Electron. 2004, 40 (10), 1511-1518.

(45) Fan, S.; Suh, W.; Joannopoulos, J. D. Temporal Coupled-Mode Theory for the Fano Resonance in Optical Resonators. J. Opt. Soc. Am. A 2003, 20 (3), 569-572.

(46) Prodan, E.; Radloff, C.; Halas, N. J.; Nordlander, P. A Hybridization Model for the Plasmon Response of Complex Nanostructures. Science 2003, 302 (5644), 419-422.

(47) Masson, J.-F.; Pelletier, L.; Collins, P. Rapid FTIR Method for Quantification of Styrene-Butadiene Type Copolymers in Bitumen. J. Appl. Polym. Sci. 2001, 79 (6), 1034-1041.

(48) Wang, S.; Chang, J.; Tsiang, R. C.-C. Infrared Studies of Thermal Oxidative Degradation of Polystyrene-Block PolybutadieneBlock-Polystyrene Thermoplastic Elastomers. Polym. Degrad. Stab. 1996, 52 (1), 51-57.

(49) Kaniappan, K.; Latha, S. Certain Investigations on the Formulation and Characterization of Polystyrene/Poly (Methyl Methacrylate) Blends. Int. J. Chemtech Res. 2011, 3 (2), 708-715.

(50) Socrates, G. Infrared and Raman Characteristic Group Frequencies: Tables and Charts; John Wiley \& Sons, 2004.

(51) Weber, K.; Nesterov, M. L.; Weiss, T.; Scherer, M.; Hentschel, M.; Vogt, J.; Huck, C.; Li, W.; Dressel, M.; Giessen, H.; et al. Wavelength Scaling in Antenna-Enhanced Infrared Spectroscopy: Toward the Far-IR and THz Region. ACS Photonics 2017, 4 (1), 4551.

(52) Palik, E. D. Handbook of Optical Constants of Solids; Academic Press, 1998. 